\title{
Finite Element Analysis of Copper Deformed By Conventional Forward Extrusion
}

\author{
Morteza Soltantabar ${ }^{1}$.Dr. A. Krishnaiah ${ }^{2}$. Ali Tari ${ }^{3}$
}

\begin{abstract}
Equal channel angular extrusion (ecae) is a novel deformation process capable of imparting a large amount of plastic strain to bulk material through the application of uniform simple shear. Ecae die geometry, material properties and process conditions influence the shear deformation behavior during extrusion that in turn governs the microstructure and mechanical properties of the extruded materials. Finite element analysis, the most appropriate technique was used to analyze the deformation behavior of extruded materials without neglecting important and realistic factors like strain hardening behavior of the material, frictional conditions and speed of the process. In this study the deformation behavior of material, dead zone/corner gap formation and strain homogeneity in friction and frictionless condition achieved in the samples during ecae were studied by using commercial finite element code abaqus/cae6.11-3. The influence of channel angles, strain hardening behavior of material and friction between the billet and die was considered for simulations. Results showed that the optimal strain homogeneity in the sample with lower dead zone formation, without involving any detrimental effects, can be achieved with channel angle of 90 degrees and outer corner angle of 10 degrees for pure copper. Keywords: Severe Plastic Deformation, Equal Channel Angular Extrusion, Ultra-Fine Grain, equivalent plastic strain, conventional extrusion
\end{abstract}

\section{Introduction}

Equal-channel angular extrusion (ECAE) is an emerging metal-processing technique developed by Segal in the former Soviet Union in the 1970s, but not widely known in the West until the 1990s(i.e., [3]). The technique is able to refine the microstructure of metals and alloys, thereby improving their strength according to the Hall-Patch relationship.

Extrusion is a process used to create objects of a fixed, cross-sectional profile. A material is pushed or drawn through a die of the desired cross-section. The two main advantages of this process over other manufacturing processes are its ability to create very complex cross-sections and work materials that are brittle, because the material only encounters compressive and shear stresses. It also forms finished parts with an excellent surface finish.

Extrusion may be continuous (theoretically producing indefinitely long material) or semi-continuous (producing many pieces). The extrusion process can be done with the material hot or cold.

In this project it is combined these two processes ECAE and Conventional Extrusion as ECAE-CE. Here it was consider the influence of some parameters on this combined process. It can be obtained high strain in this kind of process.

ECAE is unique because significant cold work can be accomplished without reduction in the cross sectional area of the deformed work piece. In conventional deformation processes like rolling, forging, extrusion, and drawing, strain is introduced by reduction in the cross sectional area. ECAE produces significant deformation strain without reducing the cross sectional area. Improved mechanical and physical properties, extreme fine grains and varying textures can therefore be obtained in the processed materials. In addition, different micro- structures and mechanical properties can also be produced by changing the strain path in the billet, which in turn is achieved by changing the orientation of the billet from one pass to the next. This is accomplished by extruding the work piece around a corner. For example, a square cross section bar of metal is forced through a channel with a 90 degree angle. The cross section of the channel is equal on entry and exit. The complex deformation of the metal as it flows around the corner produces very high strain. Because the cross section remains the same, a work piece can be extruded multiple times with each pass introducing additional strain (i.e., [2]).

The die used for the ECAE consists of two channels of equal cross-sections intersecting at an angle of

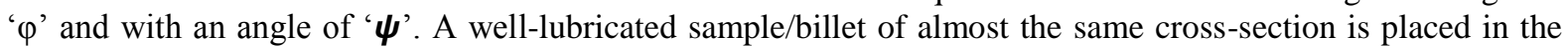
first channel and is extruded by a punch into the second channel. Under these conditions the billet moves inside the channels as a rigid body, and deformation is achieved by simple shear at the crossing plane of the channels. In this way the complete billet (except small end regions) is deformed in a uniform manner. The ECAE-CE die/ channel angle ' $\varphi$ ' $90^{\circ}$ and the ' $\boldsymbol{\psi}=0$ ' varies from $\alpha=30,40,45$ degrees. 
Physical, finite element modelling techniques have been used to study the influence of $\varphi$ and $\boldsymbol{\psi}$ on ECAE-CE process (i.e., [5,6-11]). The FEA results on aluminium alloy showed that the channel angle, ' $\varphi$ ' equal to $90^{\circ}$ is essential to achieve ultrafine microstructure with equated grains, separated by high angle grain boundaries in minimum number of passes (i.e., [6]). At the same time the physical and finite element modelling of ECAE-CE with $(\varphi=90$ and $\psi=0)$ showed non-uniform deformation in the processed material, dead metal zone formation and generation of excessive die stresses (i.e.,[4,5,7-8]). This non-uniform deformation is also observed with $\varphi>$ 90 [8,9]. Eq. (1) shows that the strain induced in the material reduces with increase in ' $\psi$ '., non-uniform deformation would occur in the material (i.e., [10-11]). The physical modelling (plasticise experiments) with $\varphi=90$ and yielded uniform deformation in the material compared to $\varphi=90$ and $\psi=0$ (i.e., [7]). The predictions of earlier studies on ' $\psi$ ' are quite contradictory, which demands a careful analysis in obtaining the optimal strain homogeneity in the deformed material

\section{Principles of ECAE-CE}

The principle of ECAE is illustrated schematically in Figure. For the die shown in Figure, the internal channel is bent through an abrupt angle, $\Phi$, and there is an additional angle, $\alpha$, which represents outer arc of curvature where the two channels intersect. The sample, in the form of a rod or bar, is machined to fit within channel and the die is placed in some form of fuss so that the sample can be extruded through the die using a plunger. The nature of the imposed deformation is simple shear which occurs as the billet passes through the die. The retention of the same cross-sectional area when processing by ECAE-CE, despite the introduction of very large strains, is the important characteristic of SPD processing and it is characteristic which distinguishes this type of processing from conventional metal-working operations such as rolling, extrusion and drawing. Since the cross-sectional area remains unchanged, the same billet may be pressed repetitively to attain exceptionally high strain (i.e.,[1]).

It consists of two orthogonal hollow cylinders intersecting at a sharp outer corner $(\boldsymbol{\psi}=0)$. The diameter of the entrance and exit channels was $20 \mathrm{~mm}$. In the second channel, the diameter was reduced from $20 \mathrm{~mm}$ to $10 \mathrm{~mm}$ and thus a reduction in area of about $80 \%$ is imposed into the material after ECAP process. This was shown in the figure 1.
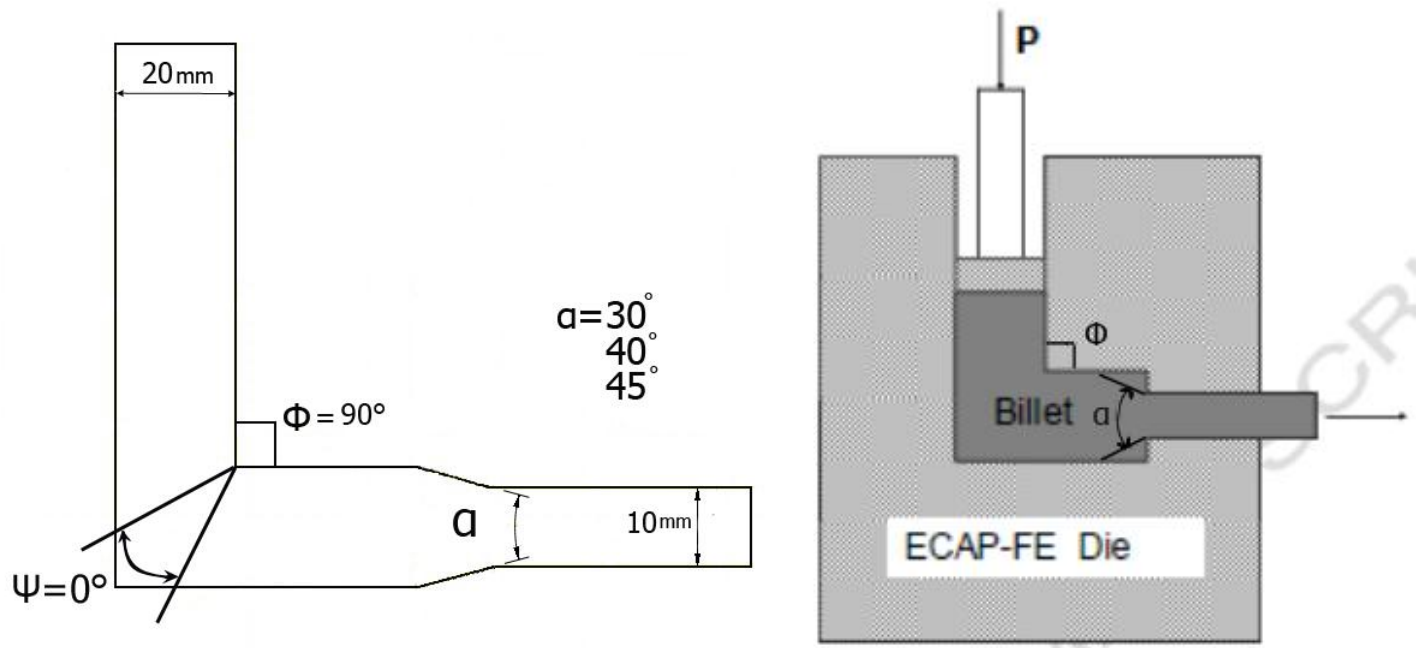

Fig1.Principal of Extrusion Channel Angular process

\section{Mechanical Properties}

It has been observed that ultra-fine grained materials produced by ECAP exhibit good hardness and high strength. It has also been observed that as grain size is reduced through the Nano scale regime, hardness typically increases Figure 2.The Hall-Petch equation expresses the grain size dependence of flow stress at any plastic strain until ductile fracturing. terms of yield stress, Hall-Petch is expressed as:

$$
\sigma_{0}=\sigma_{1}+\frac{k}{\sqrt{D}}
$$

Where $\sigma 0$ is the yield stress, $\sigma 1$ is the frictional stress opposing the dislocation motion, $\mathrm{K}$ is the Hall-Petch slope and $\mathrm{D}$ is the grain diameter (i.e.,[13]). A similar equation is obtained for hardness:

$$
\mathbf{H}_{\mathbf{0}}=\mathbf{H}_{\mathbf{1}}+\frac{\mathbf{K}}{\sqrt{\mathbf{D}}}
$$




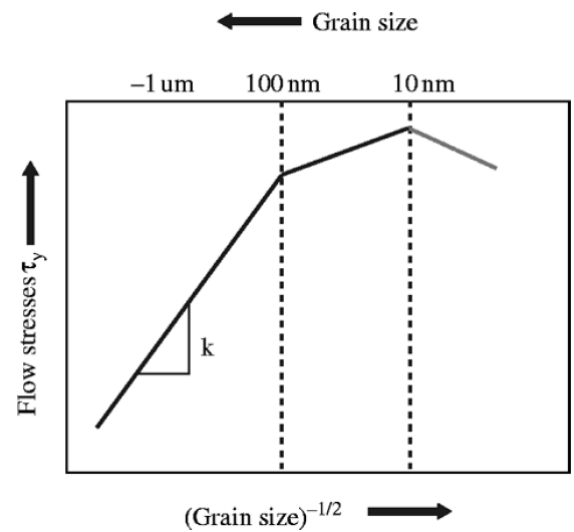

Fig2. Graphical representation of the variation in yield stress as a function of grain size in ultra-fine grained materials. Source: Sanusi and Oliver [14].

\section{Finite Element Analysis}

The discrepancy on the cangle ' $\alpha$ ' in obtaining optimal strain homogeneity in the deformed samples was addressed in the present work with the help of FEA. The analysis was carried out on $\varphi=90^{\circ}$, and $\psi=0^{\circ}$ by channel angle $(\varphi) 90$ degrees in frictionless condition, for a specific die geometry $\left(\varphi=90^{\circ}\right.$ and $\left.\alpha=30^{\circ}\right)$ also has been applied two different friction coefficient $(\mu=0,0.1)$. In contrast to the different and single ' $\alpha$ ' angles considered in the earlier works (i.e., [4,5,6-11]). The commercial finite element code, Abaqus/CAE 6.11-3, was used to carry out the simulations. Die was simulated in analytical rigid which has been fixed on reference point, reference point was demonstrated by rotated cross on lower wall of the die. In addition deformable billet by dimensions $100 \times 20 \mathrm{~mm}^{2}$ was modeled for 2D plane strain simulations with elastic-plastic material. Pure copper was applied in this simulation and following table indicates the properties of before mentioned metal

\begin{tabular}{ll}
\hline Material parameters & Value \\
\hline Young's modulus $(\boldsymbol{E})$ & $120 \mathrm{GPa}$ \\
\hline Poisson's ration $(\boldsymbol{v})$ & 0.34 \\
\hline Density $(\boldsymbol{\rho})$ & $8.90 \mathrm{~g} / \mathrm{cm}^{3}$ \\
\hline Strain hardening exponent $(\boldsymbol{n})$ & 0.3144 \\
\hline Strength coefficient $(\boldsymbol{K})$ & $359 \mathrm{MPa}$ \\
\hline
\end{tabular}

Table 1 Copper properties has been considered for simulations

All simulations were performed with a speed of $100 \mathrm{~mm} / \mathrm{s}$ on top of the billet, which generates negligible heat, due to the plastic deformation. CPE4R (A 4-node bilinear plane strain quadrilateral, reduced integration, hourglass control) elements for billet, rigid elements for die and punch were used (i.e.,[12]). Adaptive meshing was used in the simulations. To avoid distortion of the mesh due to sharp inner corner, a small fillet radius was created on the die. The simulations in this study were carried out with a refined mesh, with an element edge size of $0.4 \mathrm{~mm}$.

\section{Influence of geometry on PEEQ}

Geometry parameters like corner angles and alpha angel can influence on PEEQ which as following articles has been mentioned their effects.

\subsection{For Different Alpha Angles}

The PEEQ values are shown at the end of the deformation process to study the strain homogeneity achieved in the fully deformed sample. The PEEQ values achieved in the sample simulated with sharp alpha angle figure 3. were mostly constant except at the outer side of the sample. The effective strains obtained were uniform across $80 \%$ of the width of the sample while rest of the width was subjected to higher strains. In practice heavy stresses are needed to generate such strains that can cause damage to the die surface and score the surface of the deformed samples. In addition, it increases the load requirements. With an increase in the alpha angle , the non-uniformity in strain distribution increased at the outer side of the samples as shown in Fig. The effective strain achieved with $\alpha=30^{\circ}$ Fig. was uniform across $95 \%$ of the width of the sample, the remaining 5\% being subjected to non-uniform deformation due to the effect of friction and dead zone at the alpha angle. With 
$\alpha=40^{\circ}$ and $45^{\circ}$. the effective strains were uniform across $75 \%$ of the width. However, the range of the strain variation in $\alpha=30^{\circ}$ was not as high as in the case of $\alpha=40^{\circ}$ and $45^{\circ}$.

When $\alpha=0^{\circ}$, the simulations resulted in considerable amount of non-uniform deformation as occurred in previous finite element and physical modelling (i.e., [4, 5, 6-8]). The results, however, are quite different from the plasticise experimental results, which showed uniform deformation with $\alpha=30^{\circ}$ (i.e.,[7]). The nonuniformity in deformation with $\alpha=40^{\circ}$, along the outer side of the sample, is the greatest of all the alpha angles studied currently. Further, non-uniform deformation was observed even with $\alpha=45^{\circ}$. Hence, it can be shown that $\alpha=30^{\circ}$ is the best angle to obtain optimum strain homogeneity in the deformed samples

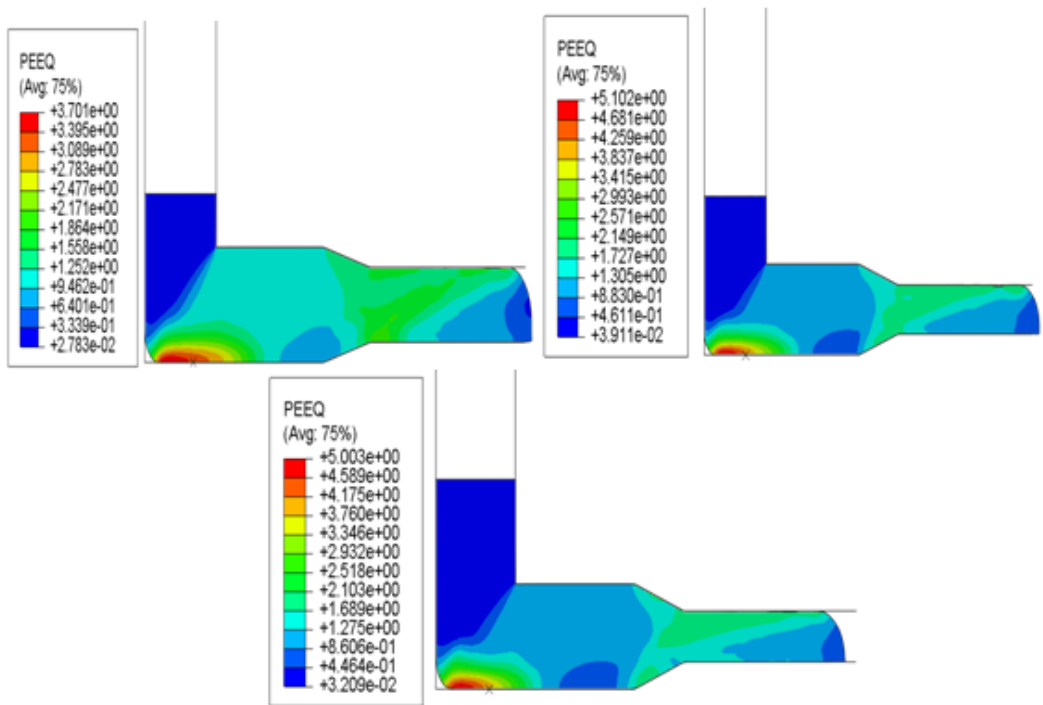

Fig3. PEEQ distribution of the samples simulated with $\varphi=90^{\circ}$ and (a) $\alpha=30^{\circ}$, (b) $\alpha=40^{\circ}$, (c) $\alpha=45^{\circ}$

Moreover for more details a diagram can be drawn according to (normalized width across (A-B)), as you can see in following diagram figure 4(a). PEEQ values for all angels for 60 percent of the width of the Chanel has equal amount of 1 .after that there is an increase for all of angles.it means that the value of PEEQ near to the bottom of the wall Chanel has maximum value amount.

As you can see in following diagramfigure 4(b) (Normalized width across (C-D)), there is a drastic decrease about 15 to 55 percent of width of sample for all alpha angles. but for $\alpha=45^{\circ}, \mu=0$ is more than others which means the region near to lower wall of the die has less effective strain comparing to other regions

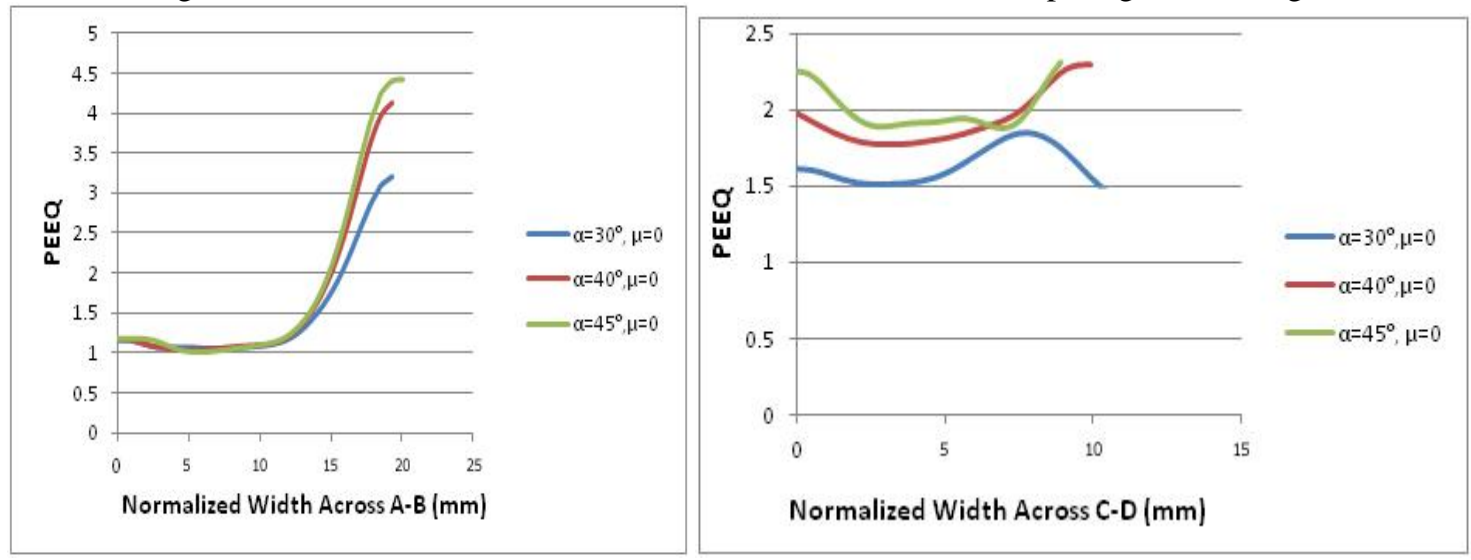

(a)

(b)

Fig4. PEEQ diagrams for different alpha angles $(\alpha),(b)$.friction 0

As you can see in following diagram figure 5. there is a drastic increase about 10 to 70 percent of width of sample $\varnothing=90^{\circ}$ which means the region near to lower wall of the die has more strain. While for other alpha angels can be observed reduction in same region. So we can conclude that more strain can be achieved by $\alpha=40^{\circ}$ $\mu=0.1$ based on this diagram .

There is drastic decrease of width of sample .for both $\alpha=40^{\circ}$ and $\alpha=45^{\circ}$ and slight decrease for $\alpha=30^{\circ}$.

so that $\alpha=30^{\circ}$ doesn't have an obvious change in PEEQ value amount throughout the procedure and slightly decrease from PEEQ 1.8 at the beginning to PEEQ 1.5 at the end . 
For $\alpha=40^{\circ}$ has a different result because its PEEQ value amount at the end of procedure is higher than its starting point.

It starts on PEEQ 2 and ends up on PEEQ 2.2 for the last angel which is $\alpha=45^{\circ}$ we have the equal PEEQ value amount both for starting point and end point which is 2.57

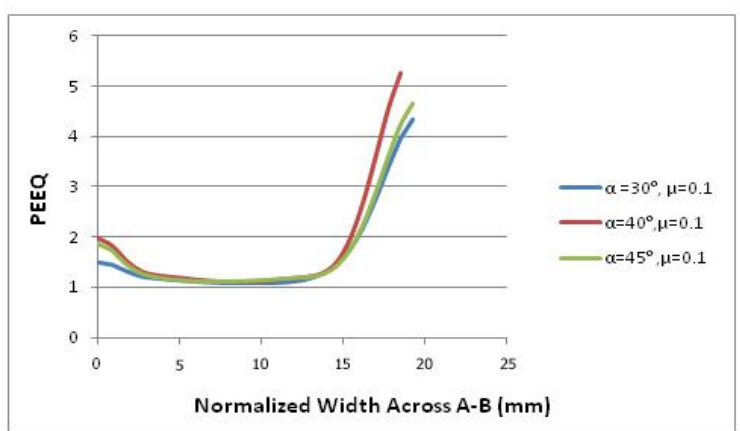

(a)

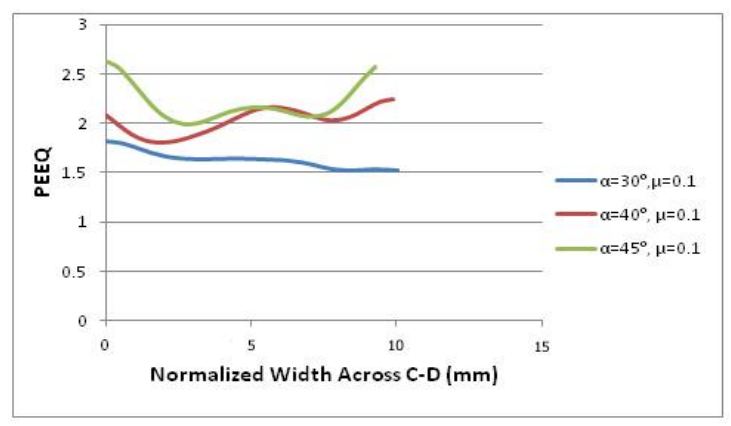

(b)

Fig5.PEEQ diagrams for different alpha angles $(\alpha)$,(b).friction 0.1

\section{Conclusion}

In the present study, FEA was carried out the deformation of copper by combined ECAE and CE. The following conclusions are drawn.

- It can be observed that the value of PEEQ in the ECAE region was increased to 4.3 for angle alpha is equal to 45 degrees and friction for zero.

- It can also observe that the value of PEEQ in CE region was increased to 2.3 for angle alpha is equal to 45 degrees and friction for zero.

\section{Reference}

[1] Vaclav Sklenicka, Jiri Dvorak, Milan Svoboda, PetrKral and Marie Kvapilova, Equal-Channel Angular Pressing and Creep in Ultrafine-Grained Aluminium and Its Alloys DOI: $10.5772 / 51242$

[2] V.M. Segal, Equal Channel Angular Extrusion: From Macromechanics to Structure Formation, Mater. Sci. Eng. A, Vol A271,[5] 1999, p 322-333

[3] V.M. Segal, V.I. Reznikov, A.E. Drobyshevskiy, V.I. Kopylov, Plastic working of metals by simple shear, Russ. Metall. 1 (1981) 99-105.

[4] Mater. 35 (1996) 143-146.

[5] H.S. Kim, M.H. Seo, I.H. Sun, On the die corner gap formation in equal channel angular pressing, Mater. Sci. Eng. 291A (2000) 86-90.

[6] H.S. Kim, Evaluation of strain rate during equal channel angular pressing, J. Mater. Res. 17 (2002) 172-179.

[7] K. Nakashima, Z. Horita, M. Nemoto, T.G. Langdon, Influence of channel angle on the development of ultrafine grains in equal channel angular pressing, Acta Metall. Mater. 46 (1998) 1589-1599

[8] Shan, I.G. Moon, H.S. Ko, J.W. Park, Direct observation of shear deformation during equal channel angular pressing of pure aluminum, Scripta Mater. 41 (1999) 353- 357

[9] V.M. Segal, Equal channel angular extrusion: from macromechanics to structure formation, Mater. Sci. Eng. 271A (1999) 322333.

[10] J.R. Bowen, A. Gholinia, S.M. Roberts, P.B. Pragnell, Analysis of the billet deformation behaviour in equal channel angular extrusion, Mater. Sci. Eng. 287A (2000) 87-99

[11] J.W. Park, J.Y. Suh, Effect of die shape on the deformation behavior in equal channel angular pressing, Metall. Mater. Trans. 32A (2001) 3007-3014.

[12] T. Altan, S.I. Oh, H.L. Gegel, Metal forming fundamentals and applications, American society for metals, Ohio, Carnes Publication Services, 1983, p. 58 and p. 237.

[13] 18. Carlton CE, Ferreira PJ. What is behind the inverse Hall-Petch effects in nanocrystalline materials? Acta Mater. 2007;55:3749. http://dx.doi. org/10.1016/j.actamat.2007.02.021

[14] Sanusi KO, Oliver GJ. Effects of grain size on mechanical properties of nanostructured copper alloy by severe plastic deformation (SPD) process. J Eng Design Technol. 2009;7(3):335-341. http://dx.doi.org/10.1108/17260530910998721 